\title{
Spectral properties of LIGNOHUMATE $®$ as affected by the duration of the technological process
}

Khundzhua D.A. ${ }^{1}$, Yakimenko O. ${ }^{2}$, Stepanov A. ${ }^{2}$, Gladkov O. ${ }^{3}$, Patsaeva S. ${ }^{1}$

${ }^{1}$ Faculty of Physics, Lomonosov Moscow State University, Moscow, Russia, dasha.ok@list.ru,

${ }^{2}$ Faculty of Soil Sciences, Lomonosov Moscow State University, Moscow, Russia

${ }^{3}$ LTD RET, St-Petersburg, Russia

Keywords: himic-like substances, absorption, fluorescence

doi: 10.36291/HIT.2019.khundzhua.118

The key factors for obtaining high-quality humic products are organic matter (OM) source and technological regimes of OM treatment. LTD RET company has developed and patented an industrial technology for the conversion of technical lignosulfonate with following synthesis of a number of humic-like biologically active substances (HLS) [1], which finally compose the commercial humic product Lignohumate ${ }^{\circledR}$ (LH). Yield, composition and biological activity of the synthesized HLS depend on the technological regimes used (alkali content, changes in temperature, pressure, oxidation rate and time) and change with the course of technological process.

The objectives of the study are: (1) to characterize the product in a number of $\mathrm{LH}$ samples taken at different times during the technological process using spectroscopic techniques with further perspective to corroborate them with chemical composition and biological activity of LH samples; (2) to reveal qualitative differences in LH spectral features as a function of time. Samples of LH were taken from the heated working mixture at certain time intervals from the start of the oxidation process: at $0,15,30,45,60$ and 120 min. Spectroscopic measurements were performed to analyze LH samples dissolved in water. Absorption spectra were measured with photometer Solar PB2201, fluorescence spectra were detected with luminescence spectrometer Solar CM2203 with several wavelengths of excitation.

Absorption and fluorescence properties of untreated lignosulfonate differ from that for other humic products [2]. However, this study revealed characteristic spectral features caused by samples treatment. Absorption spectra of all the samples demonstrated a local maximum peak at $285 \mathrm{~nm}$ and a shoulder at $235 \mathrm{~nm}$, which indicated the presence of phenolic compounds. The intensity of peak at $285 \mathrm{~nm}$ in absorption spectra was apparently decreasing along with duration of the technological process. Fluorescence spectra of all samples upon excitation at 235 or $270 \mathrm{~nm}$ showed two overlapping emission bands: with maximum at $320 \mathrm{~nm}$ (phenolic fluorescence) and within $400 \ldots 420 \mathrm{~nm}$ (humiclike fluorescence). The intensities ratio of those bands has been drastically changed during technological processing, after 15 min of treatment the UV emission band peaked at 320 $\mathrm{nm}$ (excited at $235 \mathrm{~nm}$ ) became prevailing in spectra and the maximum of the blue band shifted from 400 to $420 \mathrm{~nm}$. Fluorescence quantum yield of treated samples was sufficiently lower for treated samples than that for untreated, and was found slightly decreasing along with duration of the process. This makes possible to distinguish untreated and treated LH samples even at short duration of process. This knowledge will help to determine the optimum duration of the process for obtaining a high-quality product.

Acknowledgements. This work was supported by the Russian Foundation for Basic Research (RFBF) under Grant Number 18-016-00078.

References

1. US Patent No. 7198805 B2. Method for Producing Humic Acid Salts. April 3, 2007.

2. Yakimenko O. et al. //J. Soils and Sediments. 2018. 18(4):1279-1291. 